\title{
Activity and Impact on Resistance Development of Two Antivirulence Fluoropyrimidine Drugs in Pseudomonas aeruginosa
}

\author{
Francesco Imperi ${ }^{1,2 *}$, Ersilia V. Fiscarelli ${ }^{3}$, Daniela Visaggio ${ }^{1}$, Livia Leoni ${ }^{1}$ and Paolo Visca ${ }^{1}$ \\ ${ }^{1}$ Department of Science, Roma Tre University, Rome, Italy, ${ }^{2}$ Laboratory affiliated to Istituto Pasteur Italia-Fondazione Cenci \\ Bolognetti, Department of Biology and Biotechnology Charles Darwin, Sapienza University of Rome, Rome, Italy, ${ }^{3}$ Laboratory \\ of Cystic Fibrosis Microbiology, Bambino Gesú Hospital, Rome, Italy
}

OPEN ACCESS

Edited by:

Natalia V. Kirienko,

Rice University, United States

Reviewed by:

Carlos Juan,

Instituto de Investigación Sanitaria de

Palma (IdISPa), Spain

Pierre Cornelis,

Vrije University Brussel, Belgium

Michael L. Vasil,

University of Colorado School of

Medicine, United States

*Correspondence:

Francesco Imperi

francesco.imperi@uniroma3.it

Specialty section:

This article was submitted to

Clinical Microbiology,

a section of the journal

Frontiers in Cellular and Infection

Microbiology

Received: 10 December 2018

Accepted: 15 February 2019

Published: 11 March 2019

Citation:

Imperi F, Fiscarelli EV, Visaggio D,

Leoni L and Visca P (2019) Activity

and Impact on Resistance

Development of Two Antivirulence

Fluoropyrimidine Drugs in

Pseudomonas aeruginosa.

Front. Cell. Infect. Microbiol. 9:49.

doi: 10.3389/fcimb.2019.00049
The rise in antibiotic resistance among bacterial pathogens has prompted the exploitation of alternative antibacterial strategies, such as antivirulence therapy. By inhibiting virulence traits, antivirulence drugs are expected to lessen pathogenicity without affecting bacterial growth, therefore avoiding the spread of resistance. However, some studies argued against this assumption, and the lack of antivirulence drugs in clinical use hampers the empirical assessment of this concept. Here we compared the mode of action and range of activity of two drugs which have been proposed for repurposing as quorum sensing and pyoverdine inhibitors in the human pathogen Pseudomonas aeruginosa: the anticancer drug 5-fluorouracil (5-FU) and the antimycotic drug 5-fluorocytosine (5-FC), respectively. The effect on bacterial growth, emergence and spread of resistance, and activity against clinical isolates were assessed. Our results confirm that 5-FU has growth inhibitory activity on reference strains and can rapidly select for spontaneous resistant mutants with loss-of-function mutations in the upp gene, responsible for uracil conversion into UMP. These mutants were also insensitive to the anti-pyoverdine effect of 5-FC. Conversely, 5-FC did not cause relevant growth inhibition, likely because of poor enzymatic conversion into 5-FU by cytosine deaminase. However, coculturing experiments showed that 5 -FU resistant mutants can outcompete sensitive cells in mixed populations, in the presence of not only 5-FU but also 5-FC. Moreover, we observed that serial passages of wild-type cells in 5-FC-containing medium leads to the appearance and spread of 5-FC insensitive sub-populations of 5-FU resistant cells. The different effect on growth of 5-FU and 5-FC was overall conserved in a large collection of cystic fibrosis (CF) isolates, corresponding to different infection stages and antibiotic resistance profiles, although high variability was observed among strains. Notably, this analysis also revealed a significant number of pyoverdine-deficient isolates, whose proportion apparently increases over the course of the CF infection. This study demonstrates that the efficacy of an antivirulence drug with no apparent effect on growth can be significantly influenced by the emergence of insensitive mutants, and highlights the importance of the assessment of resistance-associated fitness cost and activity on clinical isolates for the development of "resistance-proof" antivirulence drugs.

Keywords: acquired resistance, antimetabolite, antivirulence drug, cystic fibrosis, Pseudomonas aeruginosa, siderophore, virulence 


\section{INTRODUCTION}

Antibiotic resistance is a serious public health concern at the global level, as an alarmingly high level of drug resistance has been reported in most common bacterial pathogens (Tommasi et al., 2015), calling for the investigation of alternative therapeutic options. In the last decades, researchers started looking at virulence factors as targets for the development of novel anti-infective drugs aimed at inhibiting pathogen-dependent host damage rather than bacterial growth (Finlay and Falkow, 1997). Such molecules are referred to as antivirulence drugs (Rasko and Sperandio, 2010).

Traditional antibiotics hit essential cellular processes that are widely conserved among bacteria (Lange et al., 2007), imposing a strong selective pressure for resistant mutants and often causing damage and/or dysbiosis in the normal microbiota. Conversely, antivirulence drugs should target virulence-related traits which are typically pathogen-specific and not strictly required for bacterial growth. Hence, they are expected to exert negligible effects on commensal bacteria and to decrease the evolution rates toward resistance (Rasko and Sperandio, 2010). However, since antivirulence drugs still have to enter the cell and/or interact with specific molecular targets to exert their inhibitory activity, the existence of mechanisms conferring resistance to antivirulence compounds is predictable and, indeed, some of them have already been documented, such as modification of the target or extrusion of the antivirulence drug by efflux pumps (Shakhnovich et al., 2007; Maeda et al., 2012). Whether these mechanisms of resistance would be positively selected during the infection remains a matter of debate (García-Contreras et al., 2016; Russo et al., 2016). It has been proposed that the spread of mutant clones resistant to antivirulence drugs could depend on the "public availability" of the targeted virulence factor in the bacterial population. While shared (secreted) virulence factors are public goods that can be used by both sensitive and resistant subpopulations, individualized (cell-associated) virulence factors are private goods that benefit only the resistant producers, thus providing a selective advantage to the resistant subpopulation (reviewed in Allen et al., 2014; Ruer et al., 2015; Maura et al., 2016). Irrespective of the specific conditions, it is however commonly accepted that the selective pressure for resistance to antivirulence agents is likely much weaker than that for resistance to antibiotics (Maura et al., 2016). Unfortunately, the lack of antivirulence drugs in human or animal therapy does not allow the verification of these theories in a clinical or veterinary setting.

The present study was aimed at gaining further insights in the field of antivirulence drug discovery by comparing the mode of action, selective pressure toward resistance and range of activity of two fluorinated pyrimidine drugs, the anticancer drug 5-fluorouracil (5-FU) and the antimycotic drug 5-fluocytosine (5-FC), which have been proposed for repurposing as quorum sensing and pyoverdine inhibitors in the opportunistic human pathogen Pseudomonas aeruginosa, respectively. The antivirulence activity of 5-FU against $P$. aeruginosa was identified when it was found to counteract uracil-mediated activation of the quorum sensing response and to repress the expression of several virulence traits, including biofilm formation (Ueda et al., 2009). The antivirulence potential of fluorinated pyrimidines was later expanded by a drug repurposing screening campaign, which identified 5-FC as a potent inhibitor of pyoverdine siderophore production, and showed that this antimycotic drug can also suppress $P$. aeruginosa lethality in a mouse model of acute lung infection (Imperi et al., 2013), in line with the crucial role of pyoverdine-mediated iron uptake and virulence in this infection model (Minandri et al., 2016). The anti- $P$. aeruginosa efficacy of fluorinated pyrimidines was also supported by an in vivo screening in the Caenorhabditis elegans infection model, that revealed antipathogenic and anti-pyoverdine activities in 5-FU, 5-FC, and 5fluorouridine (Kirienko et al., 2016). Notably, 5-FU had a broad inhibitory effect on several virulence phenotypes (Ueda et al., 2009), while 5-FC appeared to exert its antivirulence activity by targeting mainly the production of the pyoverdine siderophore and of pyoverdine-regulated virulence factors (Imperi et al., 2013; Kirienko et al., 2016). These works also reported that, while 5-FC does not affect $P$. aeruginosa growth even at high concentrations, 5-FU has a strong bacteriostatic effect on $P$. aeruginosa, in agreement with an older report describing the growth inhibitory activity of 5-FU, but not of 5-FC, against this bacterium (West, 1986).

Here we provide evidence that 5-FC and 5-FU likely share the same mechanism of action, and that the modest growth inhibitory activity of 5-FC is due to poor uptake and/or limited conversion into 5-FU by $P$. aeruginosa cytosine deaminase. By using co-culturing approaches and in vitro evolution experiments, we also demonstrated that $5-\mathrm{FC} / 5-\mathrm{FU}$ insensitive spontaneous mutants with a defective pyrimidine salvage pathway readily emerge and spread in 5-FU treated populations and that, unexpectedly, these resistant mutants are also selected by 5-FC treatment, though at lower frequency. Finally, we found that the growth inhibitory and/or antipyoverdine activities of these two drugs are overall conserved in a large collection of cystic fibrosis (CF) isolates, although some inter-strain variability was observed.

\section{MATERIALS AND METHODS}

\section{Bacterial Strains, Growth Conditions, And Plasmids}

Laboratory bacterial strains and plasmids used in this study are listed in Supplementary Table 1, while the $100 P$. aeruginosa $\mathrm{CF}$ isolates analyzed in this work are described in Supplementary Table 2. The CF isolates belong to the collection of bacterial strains isolated from respiratory secretions (sputum, hypopharyngeal aspirate, bronchoalveolar lavage) of CF patients in follow-up at the Cystic Fibrosis Center of the Bambino Gesù Children's Hospital (Rome, Italy). Strain isolation and characterization were performed with the informed consent of the patients or of their parents/legal guardians for minors. Strains were grown in Lysogeny Broth, Lennox formulation (LB), or Mueller-Hinton (MH) as iron-rich media (Acumedia). The iron-depleted complex medium TSBD (Ohman et al., 1980) or the M9 minimal medium supplemented with $20 \mathrm{mM}$ sodium 
succinate (SM9, Sambrook et al., 1989) were used as iron-poor media, to which $\mathrm{FeCl}_{3}$ was added at the indicated concentrations when required. Growth and pyoverdine assays were performed on bacteria cultured at $37^{\circ} \mathrm{C}$ in 96 -well microtiter plates $(200$ $\mu l$ of medium in each well) under static conditions, unless otherwise stated.

\section{Plasmid Construction}

The plasmid pUCPupp was generated by cloning the coding sequence and the promoter region of the upp gene, previously amplified using the PAO1 genomic DNA as the template, into pUCP18. Primers and restriction sites used for PCR and gene cloning are listed in Supplementary Table 1.

The plasmid pUCP $\operatorname{cod} A \operatorname{cod} B$ was generated by extracting the coding sequence of $\operatorname{cod} A$ from plasmid pUCP $\operatorname{cod} A$ by BamHIHindIII restriction. The fragment was blunt subcloned into pUCP18codB previously digested by SmaI. The correct fragment orientation was verified by restriction analysis. All constructs were verified by DNA sequencing.

\section{Growth and Pyoverdine Measurements}

Growth was measured as the $\mathrm{OD}_{600}$ of bacterial cultures or of appropriate dilutions in sterile growth medium in a spectrophotometer or, when indicated, in a microtiter plate reader (Victor ${ }^{2} \mathrm{~V}$, Wallac). Unless otherwise stated, for growth and compound sensitivity assays bacteria were inoculated at $\sim 10^{6}$ cells $/ \mathrm{ml}$ from late exponential or early stationary phase cultures. In long-term resistance development assays, at each passage bacterial cultures were diluted 1:100 in fresh medium.

Pyoverdine production was measured as the $\mathrm{OD}_{405}$ of culture supernatants appropriately diluted in $0.1 \mathrm{M}$ Tris- $\mathrm{HCl}$ ( $\mathrm{pH} 8)$, using the supernatant of a pyoverdine-deficient mutant $\left(\mathrm{PAO} 1 \Delta p v d A\right.$ ) as blank, and normalized to the $\mathrm{OD}_{600}$ of the corresponding culture (Imperi et al., 2010). Pyoverdine production by CF isolates was assessed fluorimetrically by recording the emission at $450 \mathrm{~nm}$ upon excitation at $400 \mathrm{~nm}$ (Imperi et al., 2009) of culture supernatants appropriately diluted in sterile growth medium, and normalized to the $\mathrm{OD}_{600}$ of the corresponding culture.

\section{Selection and Characterization of Spontaneous 5-FU Resistant Isolates}

To obtain spontaneous 5-FU resistant isolates, $P$. aeruginosa PAO1 was cultured in LB until the late-exponential phase, normalized at ca. $2 \times 10^{9}$ cells $/ \mathrm{ml}$ in saline and $100-\mu \mathrm{l}$ aliquots were plated onto SM9 agar plates containing $120 \mu \mathrm{g} / \mathrm{ml} 5-\mathrm{FU}$. Five microliters of serial 10-fold dilutions of the stock bacterial suspension were also spotted onto SM9 agar in the absence of 5-FU. Frequency of spontaneous 5-FU resistant mutants was calculated as the ratio between colony forming units (CFU)/ml obtained on 5 -FU-containing plates and $\mathrm{CFU} / \mathrm{ml}$ obtained on plates without 5 -FU after $48 \mathrm{~h}$ of incubation at $37^{\circ} \mathrm{C}$.

To check the DNA sequence of the upp gene in selected 5FU-resistant mutants, their genomic DNA was extracted using GenElute Bacterial Genomic DNA kit (Sigma-Aldrich) and used as the template for a PCR with primers external to the upp gene
(Supplementary Table 1), which were also used for sequencing of both DNA strands.

\section{Statistical Analyses}

Statistical analysis was performed with the software GraphPad Instat, using either using One-Way Analysis of Variance (ANOVA) followed by Tukey-Kramer multiple comparison tests or the Kruskal-Wallis test followed by uncorrected Dunn's multiple comparison test.

\section{RESULTS}

\section{Both 5-FC and 5-FU Reduce Pyoverdine Production but Only 5-FU Causes Relevant Growth Inhibition}

As a first attempt to compare the activity of the two antimetabolite drugs 5-FC and 5-FU we assessed their effect on growth and pyoverdine production of the reference strain $P$. aeruginosa PAO1 in the iron-poor complex medium TSBD which was originally used by our group in the screening of anti-pyoverdine activity among FDA-approved drugs (Imperi et al., 2013). In line with the results recently obtained by the Kirienko's group using NGM (nematode growth medium) (Kirienko et al., 2016), we confirmed that 5-FC has poor growth inhibitory activity at concentrations up to $1 \mathrm{mM}$, while 5-FU strongly inhibits $P$. aeruginosa PAO1 growth between 100 and 1,000 $\mu \mathrm{M}$ (Figure 1). Both compounds caused strong reduction of pyoverdine production at all concentrations tested ( $\geq 10 \mu \mathrm{M}$; Figure 1).

To confirm that the differential effect of 5-FC and 5-FU on $P$. aeruginosa $\mathrm{PAO} 1$ growth was not specific to the ironpoor culture medium used to monitor pyoverdine production, we also performed growth inhibition assays in different media, including complex iron-rich media ( $\mathrm{MH}$ and $\mathrm{LB}$ ), the minimal medium SM9 and, as control, TSBD. Growth was measured in a microtiter plate reader after $20 \mathrm{~h}$ at $37^{\circ} \mathrm{C}$ under static conditions, in order to determine $\mathrm{IC}_{90}$ values (compound concentrations needed to cause $90 \%$ growth inhibition). As shown in Supplementary Figure 1, 5-FC did not cause relevant alteration of $P$. aeruginosa PAO1 growth in any tested medium. In contrast, 5-FU inhibited growth in all media, although the inhibitory effect varied depending on the medium, with $\mathrm{IC}_{90}$ values ranging from $20 \mu \mathrm{M}$ in the minimal medium SM9 to $625-2,500 \mu \mathrm{M}$ in complex media (Supplementary Figure 1).

\section{Poor Conversion of 5-FC Into 5-FU Is Responsible for the Lack of Growth-Inhibitory Activity Of 5-FC}

We have previously demonstrated that the anti-pyoverdine activity of 5-FC relies on its metabolic conversion into 5-FU in the cytoplasm of $P$. aeruginosa cells, which requires the concomitant activity of cytosine permease CodB and cytosine deaminase CodA, involved in 5-FC transport and conversion into 5-FU, respectively (Imperi et al., 2013). The finding that, differently from 5-FU, 5-FC has marginal (if any) activity on growth (Figure 1; Kirienko et al., 2016) suggests that the uptake 

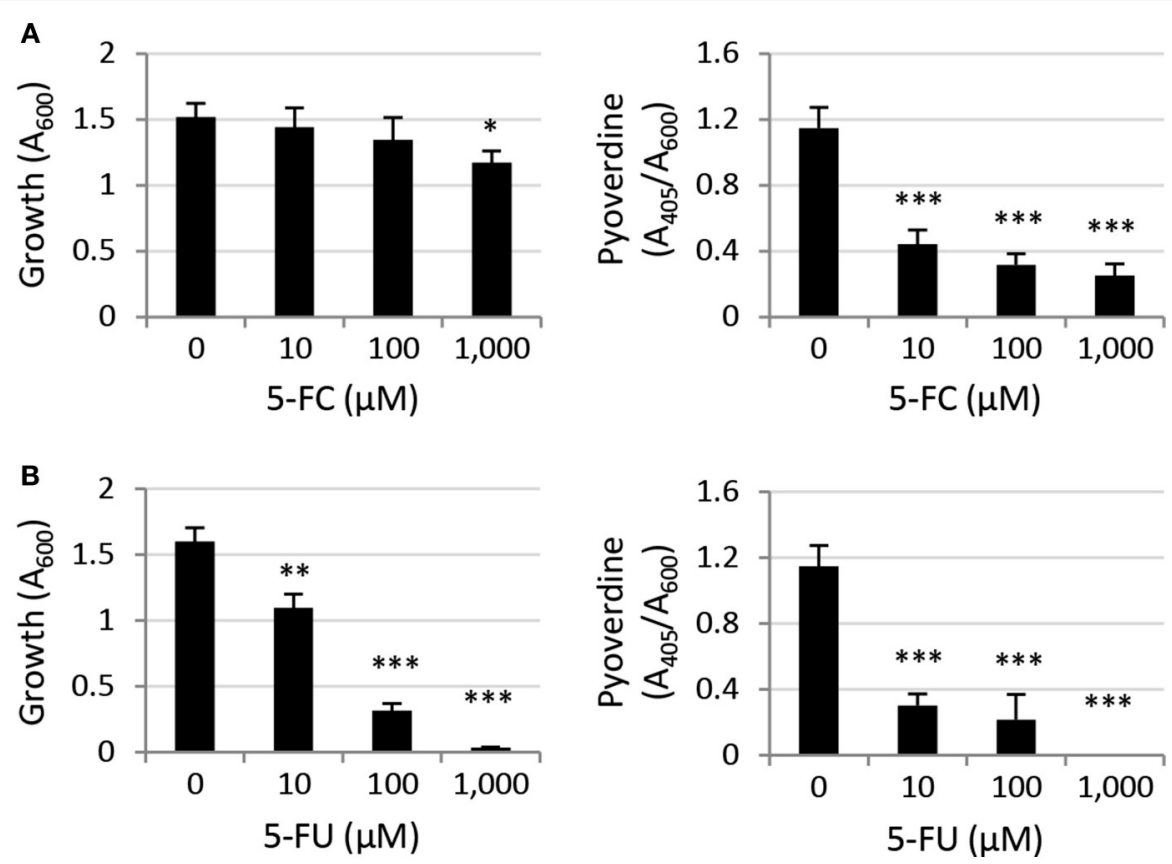

FIGURE 1 | Effect of (A) 5-fluorocytosine (5-FC) and (B) 5-fluorouracil (5-FU) (0-1,000 $\mu$ M) on P. aeruginosa PAO1 growth (left panels) and relative pyoverdine production (right panels) after 14 -h growth at $37^{\circ} \mathrm{C}$ in the iron-poor complex medium TSBD. Values represent the mean ( $\pm \mathrm{SD}$ ) of six independent assays. Asterisks indicate statistically significant differences with respect to the untreated control $\left({ }^{\star} P<0.05,{ }^{\star \star} P<0.01\right.$, ${ }^{\star \star \star} P<0.001$; ANOVA).

of 5-FC and/or its conversion into 5-FU could represent the limiting step(s) for its antibacterial activity. To explore this hypothesis, we investigated the effect of CodAB overexpression on 5-FC sensitivity in the wild type PAO1. As reported in Figure 2A, CodAB-overexpressing cells showed a marked sensitivity to 5-FC, which completely inhibited bacterial growth in SM9 minimal medium at low $\mu \mathrm{M}$ concentrations. As expected, $\mathrm{Cod} \mathrm{AB}$ overexpression did not influence the activity of 5-FU (Figure 2A), indicating that the observed increase in the growth inhibitory effect of 5-FC was not related to a general increase in sensitivity to fluorinated pyrimidine analogs. Notably, individual overexpression of CodA and, to a lesser extent, of CodB also increased $P$. aeruginosa sensitivity to 5-FC, even if the effect was much lower than that caused by $\operatorname{CodAB}$ overexpression (Figure 2B). These results indirectly imply that exogenously provided 5-FC is poorly converted into 5-FU by $P$. aeruginosa PAO1 cells, hence suggesting that the differential antivirulence and growth inhibitory effect of 5-FU is likely dependent on the intracellular concentration of this antimetabolite.

\section{Spontaneous 5-FU Resistant Mutants Acquire Mutations in the upp Gene and Are Also Insensitive to the Anti-pyoverdine Activity Of 5-FC}

Taking advantage of the strong inhibitory effect of 5-FU on $P$. aeruginosa PAO1 growth in the minimal medium SM9 (Supplementary Figure 1), we used SM9 agar plates supplemented with $120 \mu \mathrm{M} 5$-FU $\left(6 \times \mathrm{IC}_{90}\right)$ to select for spontaneous mutants resistant to this drug. In five independent experiments, we obtained 5-FU resistant isolates with an average frequency of $1.3( \pm 0.7) \times 10^{-7}$. When streaked on SM9 agar plates containing either $100 \mu \mathrm{M} 5$-FC or $100 \mu \mathrm{M} 5$-FU, all colonies appeared to be not only resistant to 5 -FU but also insensitive to the inhibitory activity of 5-FC on pyoverdine production, which was visually assessed as fluorescence under the UV light (data not shown; Visaggio et al., 2015). To confirm this qualitative observation, we selected five spontaneous mutants (obtained in independent assays) and quantitatively assessed their growth and pyoverdine production profile in the presence of high concentrations of 5-FU or 5-FC (100 and $1,000 \mu \mathrm{M})$ (Figure 3). All mutants were found to be resistant to the growth inhibitory effect of 5-FU as well as to the anti-pyoverdine activity of both drugs, although pyoverdine production was still partly repressed by $5-\mathrm{FU}$ in the spontaneous mutants $\mathrm{R} 2$ and R4 (Figure 3).

Very recently it has been shown that $P$. aeruginosa mutants resistant to 5-FC spontaneously arise after long-term treatment with 5-FC in human serum, and whole genome sequencing revealed that these mutants invariably carried mutations in the upp gene (Rezzoagli et al., 2018), which encodes the uracil phosphoribosyltransferase responsible for conversion of uracil into the nucleotide precursor UMP in the pyrimidine salvage pathway (Beck and O'Donovan, 2008). To verify whether upp mutations were also present in our spontaneous 5-FU resistant mutants, we sequenced the entire upp gene, including its promoter. A nonsense point mutation was identified in the isolate $\mathrm{R} 5$, causing premature translation termination at 

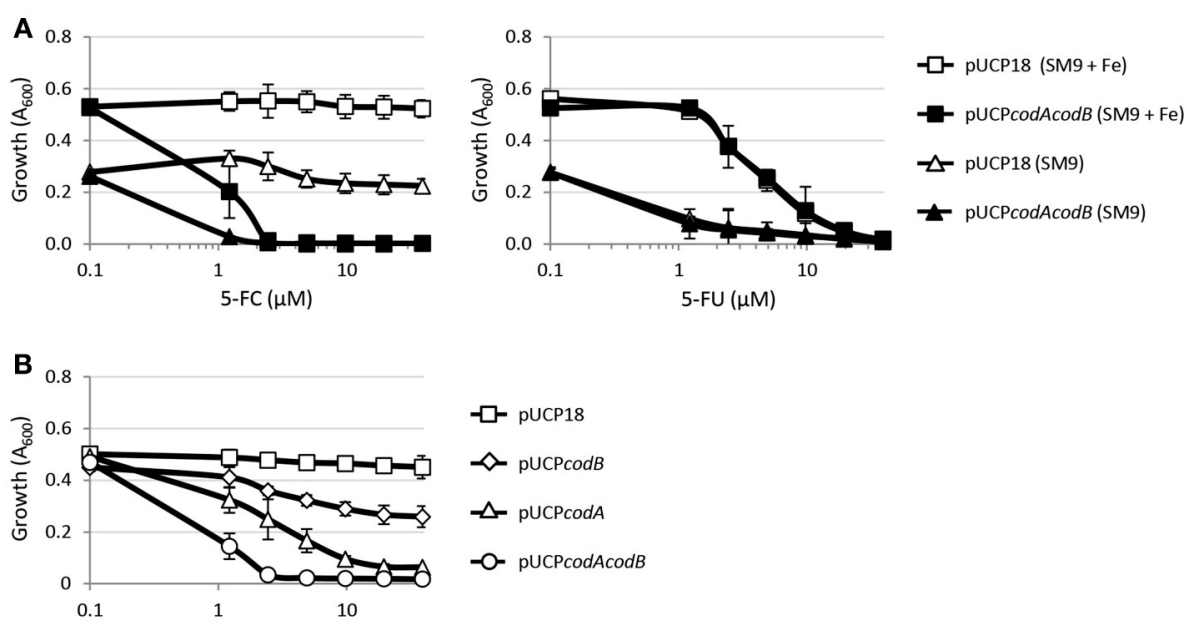

FIGURE 2 | (A) Effect of 5-fluorocytosine (5-FC, left panel) or 5-fluorouracil (5-FU, right panel) at the indicated concentrations on the growth of P. aeruginosa PAO1 pUCP18 or P. aeruginosa PAO1 pUCPcodAcodB in SM9 minimal medium, supplemented or not with $100 \mu \mathrm{M} \mathrm{FeCl} 3$ (+ Fe), after 18-h incubation in microtiter plates at $37^{\circ} \mathrm{C}$. (B) Effect of $5-\mathrm{FC}$ on the growth of $P$. aeruginosa PAO1 harboring pUCP18, pUCPCodA, pUCPcodB, or pUCPCodAcodB in SM9 with $100 \mu \mathrm{M}$ FeCl 3 after $18-\mathrm{h}$ incubation in microtiter plates at $37^{\circ} \mathrm{C}$. Growth $\left(\mathrm{OD}_{600}\right)$ was measured in a microtiter plate reader, and values represent the mean ( $\pm \mathrm{SD}$ ) of three independent assays.

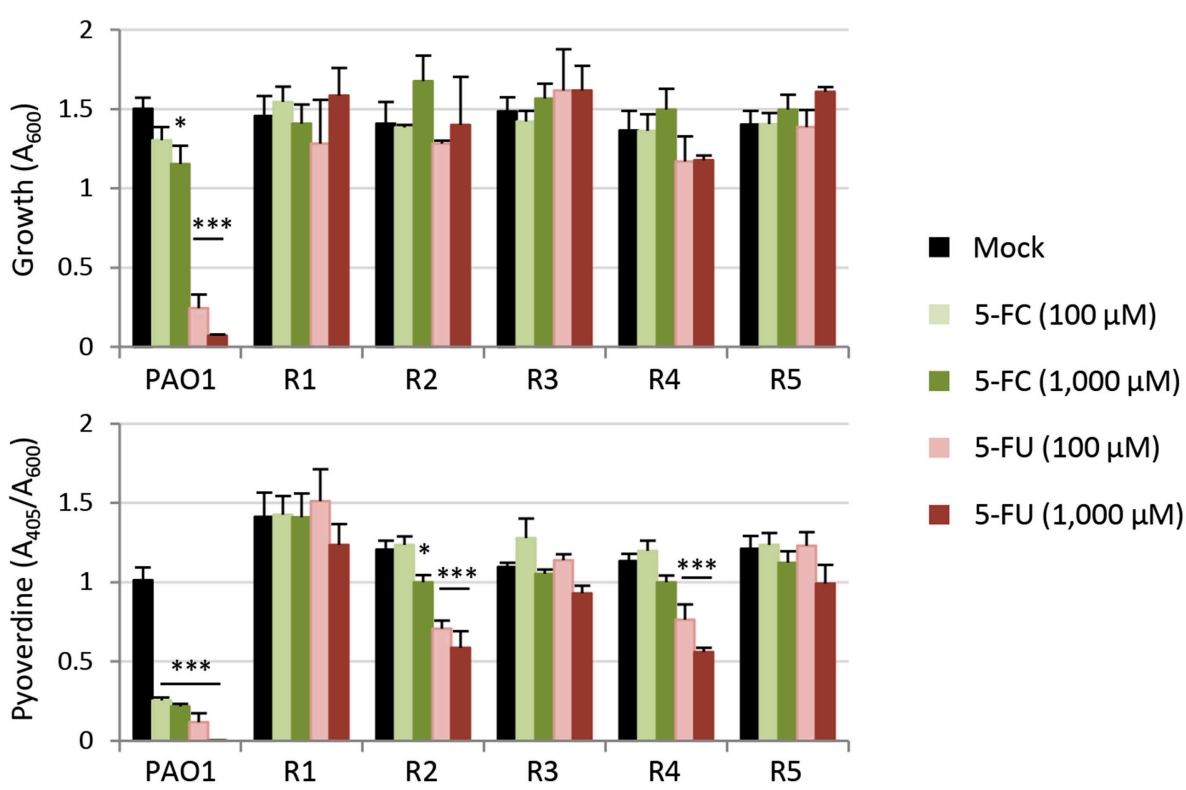

FIGURE 3 | Effect of 5-fluorocytosine (5-FC) and 5-fluorouracil (5-FU) (0-1,000 $\mu \mathrm{M})$ on growth and relative pyoverdine production (upper and lower panel, respectively) of $P$. aeruginosa PAO1 and five isogenic spontaneous mutants resistant to 5-FU (R1-R5) cultured in TSBD for $14 \mathrm{~h}$ in microtiter plates at $37^{\circ} \mathrm{C}$. Values represent the mean $( \pm S D)$ of three independent experiments. Asterisks indicate statistically significant differences with respect to the corresponding untreated contro $\left({ }^{\star} P<0.05,{ }^{\star \star *} P<0.001 ;\right.$ ANOVA $)$

codon 57 (of 213). Missense point mutations were identified in isolates R1, R2, and R4, leading to the amino acid substitution H173P (R1) or H8P (R2 and R4, carrying an identical mutation although they were selected in independent assays) (Supplementary Table 1). For the remaining clone (R3), several attempts to amplify the upp gene with different primer pairs failed, and amplification and sequencing of the $u p p$ genomic locus revealed a ca. 5-kb deletion event involving the upp gene and the neighboring genes PA4642-PA4645, uraA, cupE1, and part of cupE2 (Supplementary Figure 2). Notably, codons 8 and 173 correspond to hotspot mutation sites that were identified in the Salmonella enterica upp gene of in vitro-selected 5-FU resistant mutants (Glaab et al., 2005), and encode for histidine residues that are highly conserved in Upp proteins from eubacterial species (Supplementary Figure 3), arguing for an important role of such residues in the functionality of the 


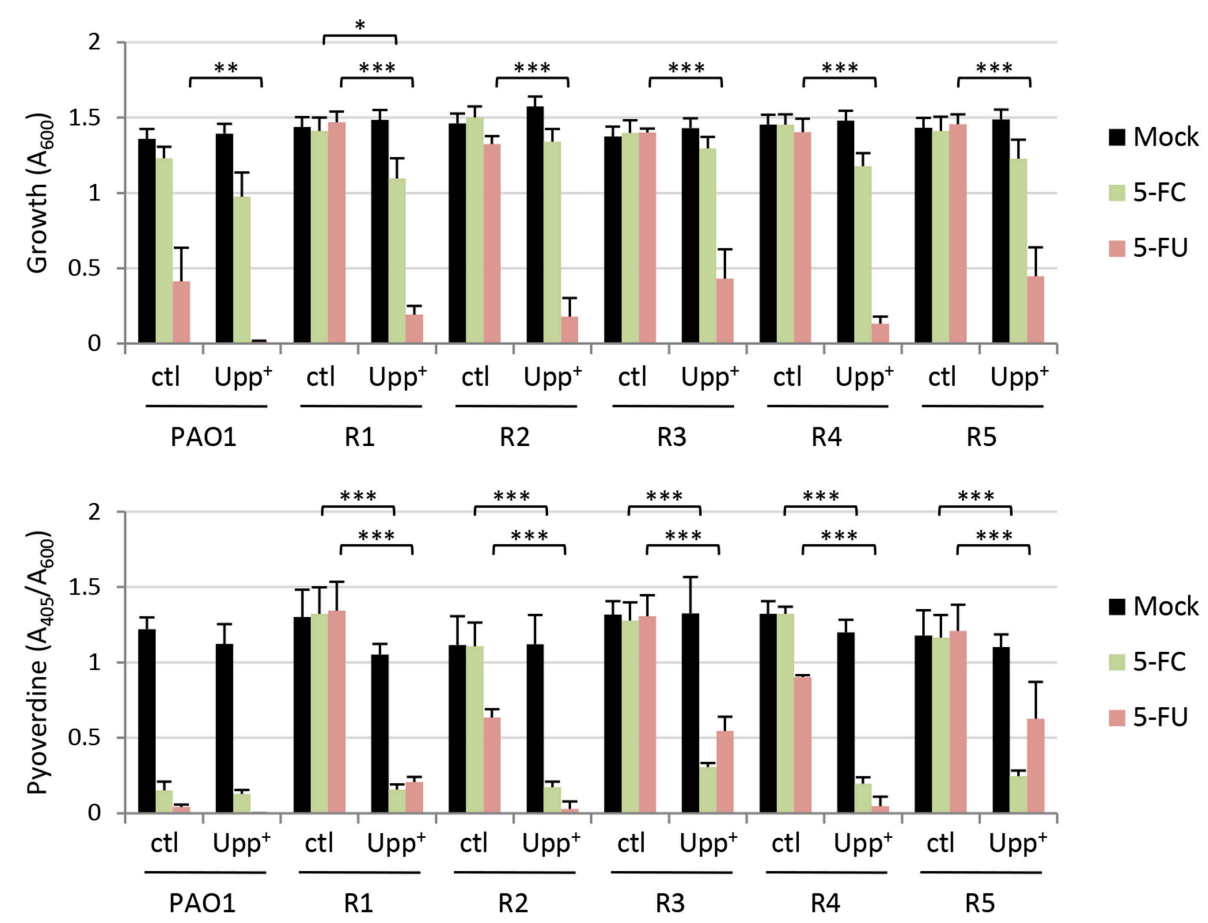

FIGURE 4 | Effect of 5-fluorocytosine (5-FC) and 5-fluorouracil (5-FU) supplemented at $100 \mu \mathrm{M}$ on growth and relative pyoverdine production (upper and lower panel, respectively) of $P$. aeruginosa PAO1 and five isogenic spontaneous mutants resistant to 5-FU (R1-R5), containing the empty plasmid pUCP18 (ctl) or the Upp-expressing construct pUCPupp (Upp $\left.{ }^{+}\right)$. Measurements were taken after 14 -h growth in TSBD in microtiter plates at $37^{\circ} \mathrm{C}$. Values represent the mean $( \pm \mathrm{SD})$ of three independent experiments. Asterisks indicate statistically significant differences with respect to the corresponding control carrying the empty plasmid and cultured under the same condition $\left({ }^{\star} P<0.05,{ }^{*} P<0.01,{ }^{\star * \star} P<0.001\right.$; ANOVA).

enzyme. To verify this hypothesis and, thus, to tentatively correlate 5-FU resistance with the loss of function of Upp, we complemented the 5-FU resistant mutants with a plasmid expressing the wild type copy of the upp gene, and determined the effect on 5-FU and 5-FC sensitivity. Overall, the expression of a functional Upp protein in 5-FU resistant mutants almost completely restored their sensitivity to 5 -FU and also rescued the anti-pyoverdine activity of 5-FC, indirectly confirming that the mutations identified in the upp gene of these isolates likely result in the loss of Upp function (Figure 4).

\section{5-FU Resistant Mutants Are Positively Selected in the Presence of Clinically-Relevant Concentrations of Both 5-FU And 5-FC}

The availability of spontaneous 5-FU resistant isolates that were also insensitive to the anti-pyoverdine effect of 5-FC gave us the opportunity to assess whether the genetic events leading to 5FC insensitivity could be selected for during 5-FC treatment. As a first attempt to investigate this issue, we co-cultured the wild type PAO1 and each spontaneous resistant isolate (R1-R5) in a 100:1 ratio in the iron-poor medium TSBD in the presence or absence of 5-FC at $400 \mu \mathrm{M}$, corresponding to the maximum serum concentration of 5-FC that is well-tolerated in humans $(50 \mu \mathrm{g} / \mathrm{mL}$, ca. $390 \mu \mathrm{M}$; Vermes et al., 2000), and a 10-fold lower concentration $(40 \mu \mathrm{M})$. When the cultures reached the stationary phase (after $\sim 10$ generations), the percentage of 5-FU resistant cells with respect to 5-FU sensitive cells was determined through serial dilution plating on SM9 agar plates containing or not $120 \mu \mathrm{M} 5$-FU. Notably, 5-FU resistant cells rose from $1 \%$ to about 10 and $15 \%$ in the presence of 40 and $400 \mu \mathrm{M} 5$-FC, respectively (Figure 5). As control, the same experiment was also performed with equivalent concentrations of 5-FU. In line with the strong growth inhibitory activity of 5-FU (Figures 1, 2; Kirienko et al., 2016), 5-FU resistant cells completely outcompeted wild type ones in the presence of both 5-FU concentrations (Figure 5). The percentage of 5-FU resistant cells remained nearly constant (ca. 1.4\%) in the absence of drugs (Figure 5), indicating that the increased fitness of 5-FU resistant cells observed in the presence of 5-FC was actually due to their competitive advantage over wild type (sensitive) cells.

The above experiment indicates that, when a relatively abundant number of 5-FC insensitive cells (1\%) are artificially introduced in the population, they are positively selected by 5 -FC treatments. To corroborate this finding in a more natural setting, a long-term experiment was performed by sequentially culturing the wild type PAO1 in TSBD containing 40 or $400 \mu \mathrm{M} 5$-FC or 5-FU for 7 passages (each involving almost 7 generations, for a total of ca. 46 generations). Growth and pyoverdine production were monitored over passages, while the percentage of 5 -FU resistant cells was determined at the end of the experiment 


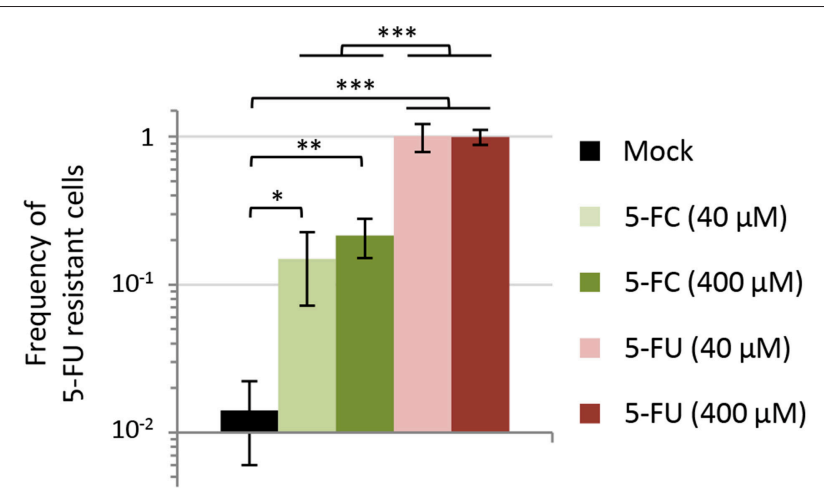

FIGURE 5 | Effect of 5-fluorocytosine (5-FC) and 5-fluorouracil (5-FU) at 40 or $400 \mu \mathrm{M}$ on the competitive fitness of spontaneous 5-FU resistant mutants of $P$. aeruginosa PAO1. Each 5-FU resistant isolate (R1, R2, R3, R4, or R5) and the parental strain PAO1 were precultured in TSBD with $50 \mu \mathrm{M} \mathrm{FeCl}_{3}$ and then inoculated at 1:1,000 final dilution in TSBD in a 1:100 ratio (5-FU R:WT). Percentage of 5 -FU resistant cells with respect to $5-\mathrm{FU}$ sensitive ones was evaluated after $16-\mathrm{h}$ incubation at $37^{\circ} \mathrm{C}$ by plating on SM9 agar plates with or without $120 \mu \mathrm{M} 5$-FU. Values represent the mean ( $\pm \mathrm{SD}$ ) of ten independent experiments (two for each 5-FU resistant isolate). Asterisks indicate statistically significant differences $\left({ }^{\star} P<0.05\right.$, ${ }^{\star \star} P<0.01$, ${ }^{\star \star \star} P<0.001$; ANOVA)

(Figure 6). This assay was aimed at verifying whether the 5FU insensitive cells emerging in the population are positively selected by the drug treatment. As expected, over the passages the cultures treated with 5-FU became progressively more resistant to this drug. After 7 passages, the cultures exposed to 5-FU showed growth and pyoverdine levels comparable to untreated cultures, irrespective of the 5-FU concentration used in subcultures (Figure 6A), and accordingly they contained only 5-FU resistant cells (Figure 6B). Conversely, sensitivity to the anti-pyoverdine effect of 5-FC remained overall constant over the course of the experiment for cultures treated with this drug, irrespective of the concentration used (Figure 6A). However, long-term exposure to 5-FC led to an 80 -fold or a 1,500-fold increase in the frequency of 5-FU resistant cells in the bacterial populations as compared to untreated cultures (Figure 6B). At the end of our assay, 5-FU resistant cells still represented $\leq 0.1 \%$ of the whole population, and for this reason they did not (yet) affect the efficacy of 5-FC in inhibiting pyoverdine production by the bacterial culture. Nevertheless, overall these results clearly indicate that, at clinically meaningful concentrations, 5-FC can exert a selective pressure in the TSBD medium which is much lower than that exerted by 5 -FU, but still enough to promote the emergence of 5-FC insensitive subpopulations.

\section{The Differential Activity of 5-FC and 5-FU Is Overall Conserved in a Collection of $P$. aeruginosa CF Isolates}

An important step in the development of any antibacterial drug is the evaluation of its activity on large panels of clinical strains. Since fluoropyridines have potential application as antivirulence adjuvants in treatment of $P$. aeruginosa infection in CF patients (Costabile et al., 2016), we compared the growth inhibitory and anti-pyoverdine effects of 5-FC and 5 -FU at two different concentrations $(10$ and $100 \mu \mathrm{M})$ on $100 P$. aeruginosa clinical strains isolated from the lung of CF patients. The collection included sequential isolates obtained during several years after establishment of the lung infection and showing different antibiotic resistance profiles (Supplementary Table 2), in order to verify whether the long-lasting in vivo adaption of $P$. aeruginosa to the CF lung and/or the acquisition of resistance to conventional antibiotics could affect the efficacy of the antivirulence drugs 5-FC and 5-FU.

Three isolates did not grow in the iron-depleted medium used to assess pyoverdine production (TSBD) (Supplementary Table 2) and, for this reason, were not included in the analysis. Moreover, a relevant percentage of CF isolates (17.5\%) did not produce detectable amounts of pyoverdine under the conditions tested, and were therefore considered as pyoverdine-deficient strains in this work (Supplementary Table 2). Notably, the percentage of these pyoverdine-deficient isolates appeared to increase over years of CF lung colonization (Supplementary Figure 4).

Although high variability in both antivirulence and growth inhibitory activities was observed among strains, CF isolates were found to be overall sensitive to the anti-pyoverdine activity of both 5-FC and 5-FU (Figure 7). However, while 5 -FC had moderate effect on the growth ( $<30 \%$ reduction) of most $(\geq 70 \%)$ isolates, and no significant differences were observed between 10 and $100 \mu \mathrm{M}$ 5-FC treatments, 5 -FU caused a strong inhibition of growth in a dosedependent manner, with $>50 \%$ growth reduction for the majority $(80 \%)$ of isolates at $100 \mu \mathrm{M}$ concentration (Figure 7 , Supplementary Table 2), in accordance with the results obtained for the reference strain PAO1 (Figure 1). For the great majority of isolates, a good correlation was observed between susceptibility to the growth inhibitory effect of 5 -FU and susceptibility to the anti-pyoverdine activity of 5-FC (Supplementary Table 2), further confirming that the modes of action of these drugs are intimately linked in $P$. aeruginosa. However, some exceptions were noted, such as isolates that were highly sensitive to the anti-pyoverdine activity of 5-FC but less susceptible to growth inhibition by 5 -FU (e.g., isolates BG17, BG79, and BG84), and isolates showing high growth inhibition but low pyoverdine reduction in the presence of 5-FU and/or 5-FC (e.g., isolates BG49 and BG55) (Supplementary Table 2).

When CF isolates were grouped according to the duration of CF lung colonization or of their antibiotic resistance profile no statistically-significant differences were observed among groups for 5-FC treatments (Supplementary Figure 5), suggesting that the adaptation of $P$. aeruginosa to the CF lung and the expression of antibiotic resistance determinants have no impact on 5-FC sensitivity. Similar results were overall obtained for 5-FU, although isolates from early stages of chronic colonization showed slightly higher sensitivity to 5-FU (Supplementary Figure 5). 

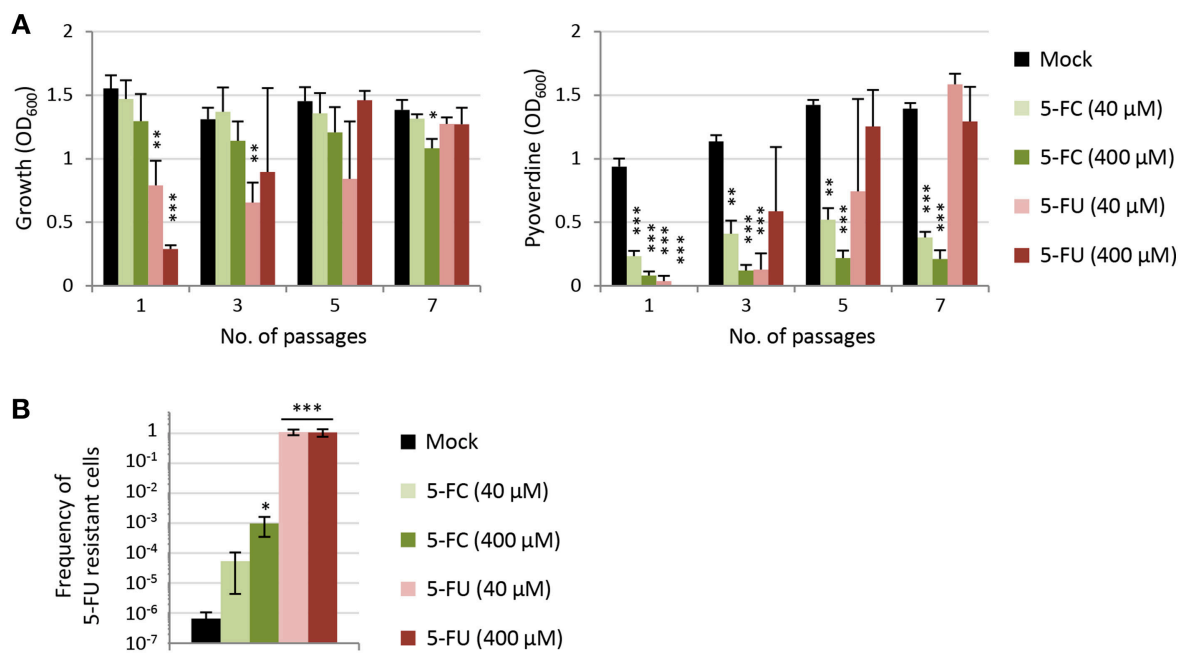

FIGURE 6 | (A) Effect of 5-fluorocytosine (5-FC) and 5-fluorouracil (5-FU) on growth and relative pyoverdine production (left and right panel, respectively) of $P$. aeruginosa PAO1 cultured for 7 subsequent passages (1:100 dilution each) in TSBD in the presence or absence of 5-FC or 5-FU at 40 or $400 \mu \mathrm{M}$. (B) Frequency of 5 -FU resistant cells after 7 passages in TSBD in the presence or in the absence of 5-FC or 5-FU at 40 or $400 \mu \mathrm{M}$. Values represent the mean ( \pm SD) of three independent experiments. Asterisks indicate statistically significant differences with respect to the corresponding untreated control ${ }^{\star} P<0.05,{ }^{\star \star} P<0.01,{ }^{\star \star \star} P<$ $0.001 ;$ ANOVA).
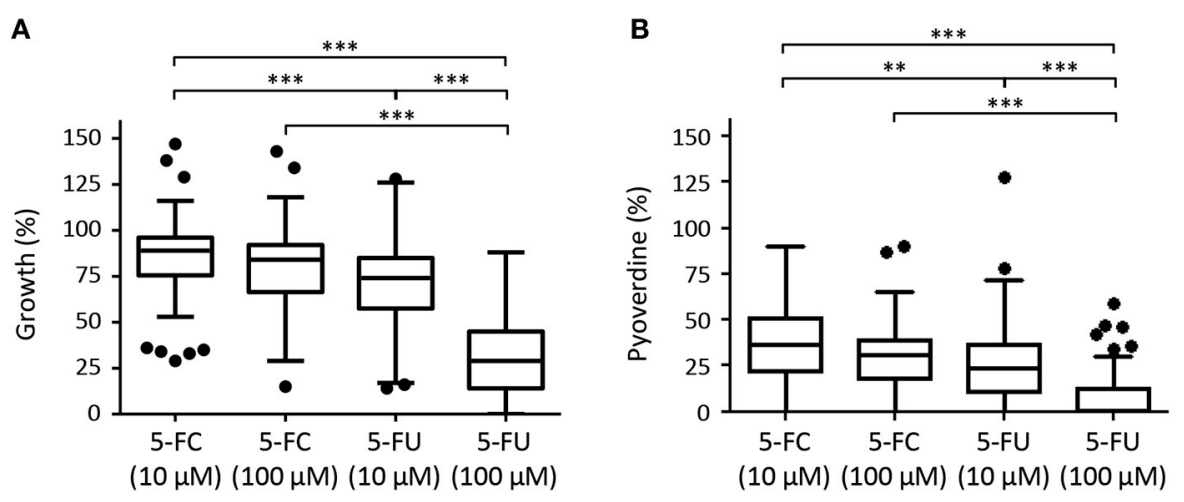

FIGURE 7 | Box plots with Turkey whiskers showing the effect of 5-fluorocytosine (5-FC) and 5-fluorouracil (5-FU) at 10 or $100 \mu \mathrm{M}$ on growth (A) and pyoverdine production (B) in a collection of 97 (A) or 80 (B) $P$. aeruginosa CF isolates cultured in TSBD until the late-exponential growth phase. Data are expressed as percentage with respect to untreated cultures (100\%). For each isolates, values are the mean of two or three independent assays. Of the 100 isolates originally included in the collection, 3 did not grow and 17 did not produce detectable amounts of pyoverdine under the conditions tested, and were not included in the plots shown in (A,B), respectively. Black dots represent the outliers. Asterisks indicate statistically significant differences $\left({ }^{\star \star} P<0.01,{ }^{\star \star \star} P<0.001\right.$; Kruskal-Wallis).

\section{DISCUSSION}

By definition, antivirulence drugs are compounds that should suppress virulence phenotypes without affecting bacterial growth. This paradigm has been used in the last 15 years to search for antivirulence activities in natural or synthetic compounds, as well as in drugs already used for other clinical purposes, for their repositioning as antivirulence agents (RangelVega et al., 2015; Mühlen and Dersch, 2016; Silva et al., 2016; Dickey et al., 2017; Johnson and Abramovitch, 2017; Rampioni et al., 2017a). Hundreds of antivirulence compounds have been proposed so far, and for most of them it has been demonstrated that they actually inhibit pathogenic traits at concentrations that have no impact on bacterial growth and cell viability, at least in vitro. The selectivity toward virulence is indispensable to ensure that antivirulence drugs provide an important advantage over traditional antibiotics, i.e., the lower selective pressure toward the emergence and spread of drug resistance. However, relatively few studies have actually explored the possible acquisition of resistance to antivirulence drugs and/or the effect of such resistance on the efficacy of antivirulence treatments.

To investigate this aspect, in this work we analyzed two highly-related antivirulence drugs endowed with significant antivirulence activity and different impact on $P$. aeruginosa growth, namely the fluorinated pyrimidine analogs 5-FU and 5-FC (Figure 1, Supplementary Figure 1; Kirienko et al., 
2016). The growth inhibitory activity of 5-FU allowed us to easily select spontaneous 5-FU-resistant mutants, which were characterized by loss-of-function mutations in the upp gene, responsible for conversion of uracil into the nucleotide precursor UMP in the pyrimidine salvage pathway (Figures 3, 4, Supplementary Table 1; Beck and O’Donovan, 2008). This implies that the growth inhibitory activity of 5-FU is mainly related to inhibition of nucleic acid synthesis, as it has been proposed to occur in yeasts and cancer cells (Vermes et al., 2000; Longley et al., 2003). Interestingly, 5-FU-resistant mutants also became resistant to the anti-pyoverdine activity of 5-FC (Figure 3). This finding is not new, as mutations in the upp gene were recently identified through whole genome sequencing in 5-FC insensitive clones of $P$. aeruginosa selected during 5-FC treatment after subsequent passages in human serum (Rezzoagli et al., 2018), a medium in which iron uptake mediated by pyoverdine is important for growth (Bonchi et al., 2015). The evidence that Upp dysfunction confers resistance to both 5-FU and 5-FC strongly suggests that these two drugs likely share the same mechanism(s) of action. Accordingly, we found that 5-FC becomes highly toxic to $P$. aeruginosa cells which ectopically over-express both CodB and CodA or CodA alone (Figure 2), implying that poor cytoplasmic conversion of 5-FC into 5 -FU is responsible for the modest growth inhibitory effect of 5-FC.

To date, the molecular target(s) and mechanism(s) of action allowing 5-FU metabolic derivatives to exert their antibacterial or anti-pyoverdine activity are still unknown. It could be hypothesized that fluorinated ribonucleotides have a different impact on the transcription and/or translation of different subsets of genes, with some virulence genes (e.g., pyoverdine genes) being more sensitive to inhibition of gene expression than housekeeping genes, thus explaining the antivirulence activity of these compounds at intracellular concentrations that do not affect growth. Such kind of effect has been proposed to explain the selective inhibition of some virulence genes by azithromycin at sub-MIC concentrations in $P$. aeruginosa (reviewed in Imperi et al., 2014). An alternative hypothesis is that fluorinated ribonucleotides could cause a general imbalance in the metabolic fluxes of the cell, which would be proportional to the intracellular concentration of the toxic compounds. At sub-inhibitory concentrations, this could result in the redistribution of cell energy and metabolites toward essential functions at the expense of non-essential processes, such as the energy-demanding biosynthesis of the pyoverdine siderophore (Visca et al., 2007), in order to ensure cell viability and reproduction. Although some reports have indeed highlighted some direct or indirect effects of catabolism and/or nutrient availability on virulence gene expression in $P$. aeruginosa (Linares et al., 2010; Yeung and Hancock, 2011; Raneri et al., 2018), further studies are still needed to verify and characterize the possible link between the metabolic state of the cell and its virulence potential.

What was unexpected is the finding that, when 5-FU/5FC resistant mutants were artificially mixed with a population of sensitive cells in a culture medium (TSBD) in which 5FC has basically no growth inhibitory effect and pyoverdine is dispensable for growth (Figure 1, Supplementary Figure 1;
Imperi et al., 2013), these mutants were positively selected in the presence of 5-FC (Figure 5). Such evidence was corroborated by subsequent passages of the wild type strain PAO1 in the presence of 5-FC, which led to the emergence and spread of small sub-populations of 5-FC insensitive cells (Figure 6). This result clearly indicates that 5-FC may exert some selective pressure for resistance, even under conditions where it has no apparent effect on growth. Whether this might be due to subtle effects of 5-FC on metabolism or to a competitive advantage of cells with an impaired pyrimidine salvage pathway ( $u p p$ mutants) over parental cells under the specific conditions tested remains to be determined. It must be remarked, however, that 5-FC/5-FU resistant mutants are much more readily selected by $5-\mathrm{FU}$ treatments than by 5-FC treatments (Figures 5, 6), in line with the relevant growth inhibition caused by 5-FU (Figure 1 and Supplementary Figure 1). This provides a direct evidence that the emergence and spread of drug insensitive populations are proportional to the growth inhibitory activity of the drug (Maura et al., 2016).

With respect to the highly-conserved and constitutivelyexpressed antibiotic targets, the presence, expression levels and relevance to infection of virulence factors are often straindependent. This is especially true for opportunistic pathogens, such as $P$. aeruginosa, in which the virulence potential is multifactorial and combinatorial, implying that different combinations of pathogenic traits are important for virulence in different strains and/or in different infection models (Lee et al., 2006; Dubern et al., 2015). Thus, an important aspect of antivirulence drug development should be the evaluation of the range of antivirulence activity on large panels of clinical isolates (García-Contreras et al., 2013; Rampioni et al., 2017a,b). As demonstrated in this work using a large collection of CF isolates, the growth and pyoverdine inhibitory effects of 5-FC and $5-\mathrm{FU}$ is overall conserved for clinical isolates (Figure 7), even if few strains revealed peculiar behaviors, such as low responsiveness to one drug and high sensitivity to the other (Supplementary Table 2). Even though these exceptions were not further investigated in this work, they suggest that the activity of 5-FC and 5-FU could be at least partly decoupled in some genetic backgrounds. Finally, it should be noted that a relatively high percentage of the CF isolates (17.5\%) were found to be defective in pyoverdine production. As expected, pyoverdine deficiency was more prevalent among isolates obtained at late stages of chronic infection (Supplementary Figure 4), in accordance with previous reports showing that $P$. aeruginosa evolves in the CF lung toward less virulent phenotypes and/or the use of iron sources alternative to pyoverdine (De Vos et al., 2001; Marvig et al., 2014, 2015). However, pyoverdine-deficient strains also represented almost $10 \%$ of CF isolates from early stages of infection (Supplementary Figure 4). Given that these isolates are likely to be insensitive to the antivirulence activity of 5-FC, this evidence suggests that the treatment with 5FC could have no (or poor) efficacy in a relevant number of CF patients.

In conclusion, this work clearly demonstrates that the lack of evident growth inhibitory activity in a putative antivirulence 
drug is not sufficient to rule out that resistance mechanisms can emerge and spread in the bacterial population, even under in vitro conditions in which the targeted virulence factor(s) is not required for growth. Although it might be argued that our results could represent a specific case related to the drugs (i.e., fluorinated pyrimidines) used in the present work, it is important to note that the number of studies proposing antimetabolites or even antibiotics at sub-MIC concentrations as potential antivirulence compounds is constantly increasing (Rangel-Vega et al., 2015; Mühlen and Dersch, 2016; Silva et al., 2016; Johnson and Abramovitch, 2017; Rampioni et al., 2017a; Soo et al., 2017; D’Angelo et al., 2018). In our opinion, the isolation of drug-insensitive spontaneous mutants, either by direct selection in the presence of high (inhibitory) drug concentrations or by means of more sophisticated culturing approaches to mimic conditions in which the targeted virulence factor(s) is important for growth (Maeda et al., 2012; Rezzoagli et al., 2018), and the assessment of the fitness of these resistant mutants during drug exposure should be mandatory tests in the antivirulence research field. This would ultimately increase the chance to identify and develop antivirulence drugs that are nearly "resistance-proof."

\section{DATA AVAILABILITY}

All datasets generated for this study are included in the manuscript and/or the supplementary files.

\section{REFERENCES}

Allen, R. C., Popat, R., Diggle, S. P., and Brown, S. P. (2014). Targeting virulence: can we make evolution-proof drugs? Nat. Rev. Microbiol. 12, 300-308. doi: $10.1038 / \mathrm{nrmicro3232}$

Beck, D. A., and O'Donovan, G. A. (2008). Pathways of pyrimidine salvage in Pseudomonas and former Pseudomonas: detection of recycling enzymes using high-performance liquid chromatography. Curr. Microbiol. 56, 162-167. doi: 10.1007/s00284-007-9050-3

Bonchi, C., Frangipani, E., Imperi, F., and Visca, P. (2015). Pyoverdine and proteases affect the response of Pseudomonas aeruginosa to gallium in human serum. Antimicrob. Agents Chemother. 59, 5641-5646. doi: 10.1128/AAC.01097-15

Costabile, G., d'Angelo, I., d'Emmanuele di Villa Bianca, R., Mitidieri, E., Pompili, B., Del Porto, P., et al. (2016). Development of inhalable hyaluronan/mannitol composite dry powders for flucytosine repositioning in local therapy of lung infections. J. Control. Release 238, 80-91. doi: 10.1016/j.jconrel.2016. 07.029

D'Angelo, F., Baldelli, V., Halliday, N., Pantalone, P., Polticelli, F., Fiscarelli, E., et al. (2018). Identification of FDA-approved drugs as antivirulence agents targeting the pqs quorum-sensing system of Pseudomonas aeruginosa. Antimicrob. Agents Chemother. 62, e01296-e01218. doi: 10.1128/AAC.01296-18

De Vos, D., De Chial, M., Cochez, C., Jansen, S., Tümmler, B., Meyer, J. M., et al. (2001). Study of pyoverdine type and production by Pseudomonas aeruginosa isolated from cystic fibrosis patients: prevalence of type II pyoverdine isolates and accumulation of pyoverdine-negative mutations. Arch. Microbiol. 175, 384-388. doi: 10.1007/s002030100278

Dickey, S. W., Cheung, G. Y. C., and Otto, M. (2017). Different drugs for bad bugs: antivirulence strategies in the age of antibiotic resistance. Nat. Rev. Drug Discov. 16, 457-471. doi: 10.1038/nrd. 2017.23

\section{AUTHOR CONTRIBUTIONS}

FI and PV conceived and designed experiments and wrote the paper. FI and DV performed the experiments. FI, EF, LL, and PV analyzed the data and contributed reagents, materials, and analysis tools. All authors read and approved the final manuscript.

\section{FUNDING}

This work was supported by the Italian Cystic Fibrosis Research Foundation (grants FFC\#10/2013 and FFC\# 17/2018), the Sapienza University of Rome (grant Ateneo 2015), the Regione Lazio (prot. 85-2017-13763) and the Grant of Excellence Departments, MIUR-Italy.

\section{ACKNOWLEDGMENTS}

We are grateful to Chiara Benvenuto for technical assistance in the screening of the CF strain collection during the preparation of her bachelor thesis.

\section{SUPPLEMENTARY MATERIAL}

The Supplementary Material for this article can be found online at: https://www.frontiersin.org/articles/10.3389/fcimb. 2019.00049/full\#supplementary-material

Dubern, J. F., Cigana, C., De Simone, M., Lazenby, J., Juhas, M., Schwager, S., et al. (2015). Integrated whole-genome screening for Pseudomonas aeruginosa virulence genes using multiple disease models reveals that pathogenicity is host specific. Environ. Microbiol. 17, 4379-4393. doi: 10.1111/1462-2920.12863

Finlay, B. B., and Falkow, S. (1997). Common themes in microbial pathogenicity revisited. Microbiol. Mol. Biol. Rev. 61, 136-169.

García-Contreras, R., Maeda, T., and Wood, T. K. (2016). Can resistance against quorum-sensing interference be selected? ISME J. 10, 4-10. doi: 10.1038 /ismej.2015.84

García-Contreras, R., Martínez-Vázquez, M., Velázquez Guadarrama, N., Villegas Pañeda, A. G., Hashimoto, T., Maeda, T., et al. (2013). Resistance to the quorum-quenching compounds brominated furanone C-30 and 5fluorouracil in Pseudomonas aeruginosa clinical isolates. Pathog. Dis. 68, 8-11. doi: 10.1111/2049-632X.12039

Glaab, W. E., Mitchell, L. S., Miller, J. E., Vlasakova, K., and Skopek, T. R. (2005). 5-fluorouracil forward mutation assay in Salmonella: determination of mutational target and spontaneous mutational spectra. Mutat. Res. 578, 238-246. doi: 10.1016/j.mrfmmm.2005.05.021

Imperi, F., Leoni, L., and Visca, P. (2014). Antivirulence activity of azithromycin in Pseudomonas aeruginosa. Front. Microbiol. 5:178. doi: $10.3389 /$ fmicb. 2014.00178

Imperi, F., Massai, F., Facchini, M., Frangipani, E., Visaggio, D., Leoni, L., et al. (2013). Repurposing the antimycotic drug flucytosine for suppression of Pseudomonas aeruginosa pathogenicity. Proc. Natl. Acad. Sci. U.S.A. 110, 7458-7463. doi: 10.1073/pnas.1222706110

Imperi, F., Tiburzi, F., Fimia, G. M., and Visca, P. (2010). Transcriptional control of the $p v d S$ iron starvation sigma factor gene by the master regulator of sulfur metabolism CysB in Pseudomonas aeruginosa. Environ. Microbiol. 12, 1630-1642. doi: 10.1111/j.1462-2920.2010.02210.x

Imperi, F., Tiburzi, F., and Visca, P. (2009). Molecular basis of pyoverdine siderophore recycling in Pseudomonas aeruginosa. Proc. Natl. Acad. Sci. U.S.A. 106, 20440-20445. doi: 10.1073/pnas.0908760106 
Johnson, B. K., and Abramovitch, R. B. (2017). Small molecules that sabotage bacterial virulence. Trends Pharmacol. Sci. 38, 339-362. doi: 10.1016/j.tips.2017.01.004

Kirienko, D. R., Revtovich, A. V., and Kirienko, N. V. (2016). A high-content, phenotypic screen identifies fluorouridine as an inhibitor of pyoverdine biosynthesis and Pseudomonas aeruginosa Virulence. mSphere 1, e00217e00216. doi: 10.1128/mSphere.00217-16

Lange, R. P., Locher, H. H., Wyss, P. C., and Then, R. L. (2007). The targets of currently used antibacterial agents: lessons for drug discovery. Curr. Pharm. Des. 13, 3140-3154. doi: 10.2174/138161207782110408

Lee, D. G., Urbach, J. M., Wu, G., Liberati, N. T., Feinbaum, R. L., Miyata, S., et al. (2006). Genomic analysis reveals that Pseudomonas aeruginosa virulence is combinatorial. Genome Biol. 7:R90. doi: 10.1186/gb-2006-7-10-r90

Linares, J. F., Moreno, R., Fajardo, A., Martínez-Solano, L., Escalante, R., Rojo, F., et al. (2010). The global regulator Crc modulates metabolism, susceptibility to antibiotics and virulence in Pseudomonas aeruginosa. Environ. Microbiol. 12, 3196-3212. doi: 10.1111/j.1462-2920.2010.02292.x

Longley, D. B., Harkin, D. P., and Johnston, P. G. (2003). 5-fluorouracil: mechanisms of action and clinical strategies. Nat. Rev. Cancer. 3, 330-338. doi: $10.1038 / \mathrm{nrc} 1074$

Maeda, T., García-Contreras, R., Pu, M., Sheng, L., Garcia, L. R., Tomás, M., et al. (2012). Quorum quenching quandary: resistance to antivirulence compounds. ISME J. 6, 493-501. doi: 10.1038/ismej.2011.122

Marvig, R. L., Damkiær, S., Khademi, S. M., Markussen, T. M., Molin, S., and Jelsbak, L. (2014). Within-host evolution of Pseudomonas aeruginosa reveals adaptation toward iron acquisition from hemoglobin. MBio 5, e00966-e00914. doi: $10.1128 / \mathrm{mBio} .00966-14$

Marvig, R. L., Sommer, L. M., Molin, S., and Johansen, H. K. (2015). Convergent evolution and adaptation of Pseudomonas aeruginosa within patients with cystic fibrosis. Nat. Genet. 47, 57-64. doi: 10.1038/ng.3148

Maura, D., Ballok, A. E., and Rahme, L. G. (2016). Considerations and caveats in anti-virulence drug development. Curr. Opin. Microbiol. 33, 41-46. doi: 10.1016/j.mib.2016.06.001

Minandri, F., Imperi, F., Frangipani, E., Bonchi, C., Visaggio, D., Facchini, M., et al. (2016). Role of iron uptake systems in Pseudomonas aeruginosa virulence and airway infection. Infect. Immun. 84, 2324-2335. doi: 10.1128/IAI.00098-16

Mühlen, S., and Dersch, P. (2016). Anti-virulence strategies to target bacterial infections. Curr. Top. Microbiol. Immunol. 398, 147-183. doi: $10.1007 / 82 \_2015 \_490$

Ohman, D. E., Sadoff, J. C., and Iglewski, B. H. (1980). Toxin A-deficient mutants of Pseudomonas aeruginosa PA103: isolation and characterization. Infect. Immun. $28,899-908$.

Rampioni, G., Pillai, C. R., Longo, F., Bondì, R., Baldelli, V., Messina, M., et al. (2017b). Effect of efflux pump inhibition on Pseudomonas aeruginosa transcriptome and virulence. Sci. Rep. 7:11392. doi: 10.1038/s41598-017-11892-9

Rampioni, G., Visca, P., Leoni, L., and Imperi, F. (2017a). Drug repurposing for antivirulence therapy against opportunistic bacterial pathogens. Emerg. Top. Life Sci. 1, 13-22. doi: 10.1042/ETLS20160018

Raneri, M., Pinatel, E., Peano, C., Rampioni, G., Leoni, L., Bianconi, I., et al. (2018). Pseudomonas aeruginosa mutants defective in glucose uptake have pleiotropic phenotype and altered virulence in non-mammal infection models. Sci. Rep. 8:16912. doi: 10.1038/s41598-018-35087-y

Rangel-Vega, A., Bernstein, L. R., Mandujano-Tinoco, E. A., García-Contreras, S. J., and García-Contreras, R. (2015). Drug repurposing as an alternative for the treatment of recalcitrant bacterial infections. Front. Microbiol. 6:282. doi: $10.3389 /$ fmicb.2015.00282

Rasko, D. A., and Sperandio, V. (2010). Anti-virulence strategies to combat bacteria-mediated disease. Nat. Rev. Drug Discov. 9, 117-128. doi: $10.1038 / \mathrm{nrd} 3013$
Rezzoagli, C., Wilson, D., Weigert, M., Wyder, S., and Kümmerli, R. (2018) Probing the evolutionary robustness of two repurposed drugs targeting iron uptake in Pseudomonas aeruginosa. Evol. Med. Public Health 2018, 246-259. doi: 10.1093/emph/eoy026

Ruer, S., Pinotsis, N., Steadman, D., Waksman, G., and Remaut, H. (2015). Virulence-targeted antibacterials: concept, promise, and susceptibility to resistance mechanisms. Chem. Biol. Drug. Des. 86, 379-399. doi: $10.1111 /$ cbdd. 12517

Russo, T. A., Spellberg, B., and Johnson, J. R. (2016). Important complexities of the antivirulence target paradigm: a novel ostensibly resistance-avoiding approach for treating infections. J. Infect. Dis. 213, 901-903. doi: 10.1093/infdis/ jiv533

Sambrook, J., Fritsch, E. F., and Maniatis, T. (1989). Molecular Cloning: a Laboratory Manual, 2nd Edn. Cold Spring Harbor, NY: Cold Spring Harbor Laboratory.

Shakhnovich, E. A., Sturtevant, D., and Mekalanos, J. J. (2007). Molecular mechanisms of virstatin resistance by non-O1/non-O139 strains of Vibrio cholerae. Mol. Microbiol. 66, 1331-1341. doi: 10.1111/j.1365-2958.2007.05984.x

Silva, L. N., Zimmer, K. R., Macedo, A. J., and Trentin, D. S. (2016). Plant natural products targeting bacterial virulence factors. Chem. Rev. 116, 9162-9236. doi: 10.1021 /acs.chemrev.6b00184

Soo, V. W., Kwan, B. W., Quezada, H., Castillo-Juárez, I., Pérez-Eretza, B., García-Contreras, S. J., et al. (2017). Repurposing of anticancer drugs for the treatment of bacterial infections. Curr. Top. Med. Chem. 17, 1157-1176. doi: $10.2174 / 1568026616666160930131737$

Tommasi, R., Brown, D. G., Walkup, G. K., Manchester, J. I., and Miller, A. A. (2015). ESKAPEing the labyrinth of antibacterial discovery. Nat. Rev. Drug Discov. 14, 529-542. doi: 10.1038/nrd4572

Ueda, A., Attila, C., Whiteley, M., and Wood, T. K. (2009). Uracil influences quorum sensing and biofilm formation in Pseudomonas aeruginosa and fluorouracil is an antagonist. Microb. Biotechnol. 2, 62-74. doi: $10.1111 / j .1751-7915.2008 .00060 . x$

Vermes, A., Guchelaar, H. J., and Dankert, J. (2000). Flucytosine: a review of its pharmacology, clinical indications, pharmacokinetics, toxicity and drug interactions. J. Antimicrob. Chemother. 46, 171-179. doi: 10.1093/jac/46.2.171

Visaggio, D., Pasqua, M., Bonchi, C., Kaever, V., Visca, P., and Imperi, F. (2015). Cell aggregation promotes pyoverdine-dependent iron uptake and virulence in Pseudomonas aeruginosa. Front. Microbiol. 6:902. doi: $10.3389 /$ fmicb. 2015.00902

Visca, P., Imperi, F., and Lamont, I. L. (2007). Pyoverdine siderophores: from biogenesis to biosignificance. Trends Microbiol. 15, 22-30. doi: 10.1016/j.tim.2006.11.004

West, T. P, and Chu, C. P. (1986). Utilization of pyrimidines and pyrimidine analogues by fluorescent pseudomonads. Microbios 47, 149-157.

Yeung, A. T, Bains, M., and Hancock, R. E. (2011). The sensor kinase CbrA is a global regulator that modulates metabolism, virulence, and antibiotic resistance in Pseudomonas aeruginosa. J. Bacteriol. 193, 918-931. doi: 10.1128/JB.00911-10

Conflict of Interest Statement: The authors declare that the research was conducted in the absence of any commercial or financial relationships that could be construed as a potential conflict of interest.

Copyright $\odot 2019$ Imperi, Fiscarelli, Visaggio, Leoni and Visca. This is an open-access article distributed under the terms of the Creative Commons Attribution License (CC $B Y)$. The use, distribution or reproduction in other forums is permitted, provided the original author(s) and the copyright owner(s) are credited and that the original publication in this journal is cited, in accordance with accepted academic practice. No use, distribution or reproduction is permitted which does not comply with these terms. 